\title{
Validation of YPAS-PT - The Yale Physical Activity Survey for Portuguese Older People
}

\author{
Maria Machado, Ciro Tavares, Vera Moniz-Pereira, Helô André, Fátima Ramalho, António Veloso, \\ Filomena Carnide
}

Faculdade de Motricidade Humana, Universidade de Lisboa, Cruz Quebrada, Portugal

Email address:

mmachado@fmh.ulisboa.pt (M. Machado)

\section{To cite this article:}

Maria Machado, Ciro Tavares, Vera Moniz-Pereira, Helô André, Fátima Ramalho, António Veloso, Filomena Carnide. Validation of YPASPT - The Yale Physical Activity Survey for Portuguese Older People. Science Journal of Public Health. Vol. 4, No. 1, 2016, pp. $72-80$. doi: $10.11648 /$ j.sjph.20160401.20

\begin{abstract}
This study aimed to translate, adapt and validate the Yale Physical Activity Survey (YPAS) to Portuguese culture in order to assess the level of physical activity in a country with one of the world's highest percentage of senior citizens. Permission was obtained from the original authors and translated according to WHO guidelines. Validation included 471 older adults (70.3\% women; $74.9 \pm 6.5$ years), covering Criterion Validity, through Spearman's rho, with a cross-reference standard, by accelerometry with 96 subjects; and Reliability comprising Internal Consistency through Cronbach's alpha with 471 subjects, and Temporal Stability through Intraclass Correlation Coefficient involving 30 subjects. YPAS-PT appeared suitable and relevant with low-to-moderate levels for all psychometric parameters. Significant correlations were found for all variables, including accelerometer data ranging from 0.201 to $0.452(\mathrm{p}<0.001)$. Internal consistency $(\alpha \geq 0.835)$ and temporal stability (ICC $>0.75$ ) demonstrated high reliability. The YPAS-PT is a valid instrument to assess Physical Activity patterns of Portuguese older adults, allowing record low-intensity activities typical of sedentary behavior, which is often excluded from other Physical Activity assessment tools. The study has concluded that the Portuguese version of YPAS (i.e., YPAS-PT) is a valid instrument to assess PA patterns of Portuguese older adults for research and social interventions.
\end{abstract}

Keywords: YPAS, Older Adults, Physical Activity, Questionnaires, Validation, Survey

\section{Introduction}

Regular physical activity (PA) produces major and extensive health benefits in older adults [1]. As a result, there is a growing interest in measuring PA in independentliving individuals to enhance the understanding of the relationship between PA and health during aging with a need for effective instruments to measure and quantify PA specifically to record low-intensity activities typical of sedentary societies [2].

Aging populations have significant public health implications for Portugal, considering it has one of the world's highest percentage of senior citizens $(19.3 \%$ of people aged 65 and above) and an old-age dependency ratio of 29.4 [3]. A national survey of activity patterns in Portugal identified that $55 \%$ and $72 \%$ of the older men and women respectively were inactive [4]. This inactivity is often associated with a decrease in strength and endurance, which has a negative impact on activities of daily living
(ADL) and therefore the quality of life in the aging process $[1,5]$.

Regular PA can be assessed using objective measures including direct calorimetry, ingestion of doubly labeled water, motion sensors (e.g., accelerometers), heart rate monitors or oxygen consumption $[2,6,7]$. However, these methods are not applicable for large-scale epidemiologic studies, due to constraints in resources, labor and cost.

Although questionnaires are not objective measures, they are the instrument of choice for epidemiologic surveys. They have a generalized applicability, are cheaper and enable researchers to easily assess and monitor increases and changes in PA in large populations [2, 7]. Despite extensive use over 40 years, questionnaires still have limited reliability and validity relative to a laboratory measure, irrespective of the questionnaire chosen [2]. These limitations may be amplified among older populations due to challenges in collecting data quantifying terms related to intensity, as this changes with age [2]. Tasks and activities 
that are predominately considered "light intensity" such as household tasks may not be adequately captured with standardized questionnaires not specific for older adults [7, 8]. Standardized tools tailored to older populations are essential to determine the level and changes in PA patterns.

There has been limited attention given to PA assessment among older adults in Portugal and no validated questionnaires for this population. The International Physical Activity Questionnaire (IPAQ) developed to measure health related-PA in adult populations [9] has been used in Portugal $[4,10]$. However studies have found that IPAQ reliability is very low when applied to older populations [11] and caution is needed when administering the IPAQ to adults aged over 65 [12].

The Yale Physical Activity Survey (YPAS) is an interviewer-administered questionnaire developed to determine the type, amount and patterning of PA/exercise in older adults [13]. Based on comparative studies between different PA questionnaires [6, 7, 14-16], the YPAS [13] (1) is one of the most detailed questionnaires available for senior subjects with previously published validity, reliability and sensitivity to change; (2) presents issues easily understood and adapted to the daily routines and lifestyle of this population; (3) allows characterization of the intensity of each activity performed, as either light, moderate or vigorous; (4) identifies temporal aspects of daily movement patterns, as well as differences in seasonal activity undertaken [8, 13, 14, 17-23] and (5) used in diverse populations across nations and cultures [8, 22-25].

Comparisons between standardized tools of YPAS and IPAQ have recommended the use of the former tool over the latter for research studies due to the robustness of its specific domains, such as housework and yard work capturing lightintensity activity often excluded from other PA assessment tools $[14,15,26]$.

This paper has two objectives, firstly validation of the YPAS to Portuguese culture (YPAS-PT) and secondly assesses the PA profile and level in Portugal, a country with one of the world's highest percentage of senior citizens.

\section{Methods}

\subsection{Study Design}

The validation process began with the translation and cultural adaptation of the original version of YPAS (i.e., permission of the authors; translation; harmonization; cognitive debriefing) in order to reach the Portuguese version: the YPAS-PT (Figure 1). The validity (criterion validity) and reliability (internal consistency and temporal stability) of the YPAS-PT were then psychometrically tested using the methodology described below.

Interviews were conducted by a team of 15 examiners, supervised by a minimum of two members of the research team. The examiners undertook a total of 51 hours of training prior to the data collection to enable greater proficiency and consistency in the conduction of interviews.
Immediately prior to data collection, participants were informed about the study, accepted to participate and signed informed consent. The Ethics Committee of Faculdade de Motricidade Humana, Universidade de Lisboa, approved the study protocol.

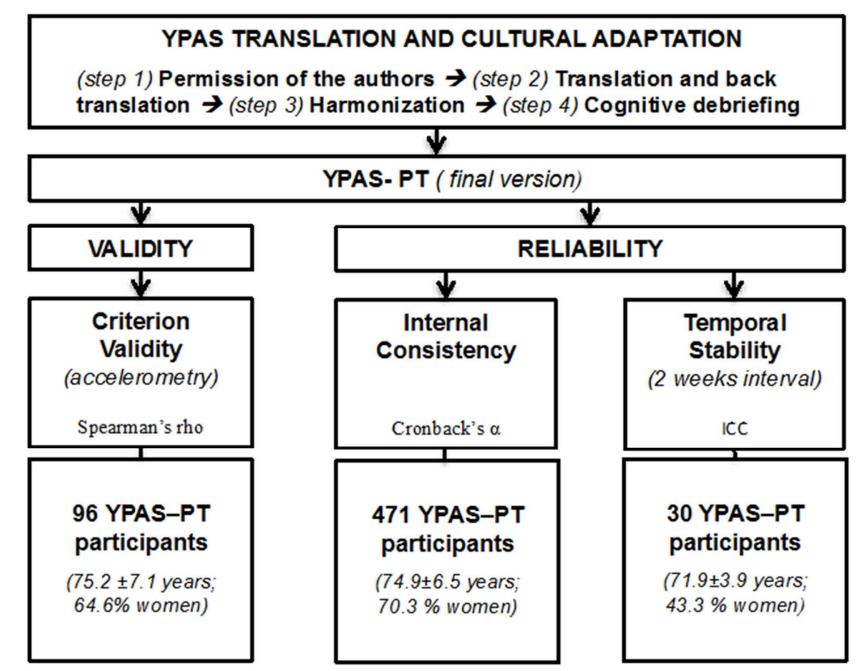

Figure 1. Study design: flowchart of the validation process of the Portuguese version of the Yale Physical Activity Survey - YPAS-PT.

\subsection{Participants}

This study has enrolled a total of 471 community-dwelling older adults, aged 65 to 96 years $(74.9 \pm 6.5$ years, $70.3 \%$ women), from the research project "Biomechanics of Locomotion in the Elderly" (PTDC/DES/72946/2006) supported by the Portuguese Foundation for Science and Technology.

Participants were randomly selected from the community and private lifestyle centers for older adults, residential aged-care facilities, senior schools, gyms and health promotion community events, from a list available on the General Directorate of Health (Ministry of Health) website within a $50 \mathrm{~km}$ radius from the Faculty laboratory. The sample recruitment allowed researchers to establish a cohort of elderly subjects from the Lisbon region from both urban and regional areas.

Inclusion criteria for participants were: aged 65 years or older and competent in speaking and comprehending the Portuguese language. Exclusion criteria included having a neurologic condition (Dementia, Parkinson or stroke); recovering from acute illness and not being able to walk independently or using a walking aid.

\subsection{The Instrument: Yale Physical Activity Survey - YPAS}

The YPAS [13] is an interviewer-administered questionnaire divided into two parts, which allows the estimation of PA of a typical week in the last month prior to evaluation. The first part, YPAS activity checklist, queries detailed information about the type, duration (h. $\left.\mathrm{wk}^{-1}\right)$ and intensity $\left(\mathrm{kcal} \cdot \mathrm{min}^{-1}\right)$ of the 25 common activities carried out by the elderly, in five domains: household, yard work, caregiving, exercise, and recreational activities. Participants report the amount of time 
spent in each activity during the mentioned week, which is then summed to provide a total PA time $\left(\mathrm{h} \cdot \mathrm{wk}^{-1}\right)$. The weekly PA energy expenditure (EE) is calculated by multiplying the total time spent in each reported activity with an intensity code $\left(\mathrm{kcal} . \mathrm{min}^{-1}\right)$ and then converted to a weekly value $\left(\mathrm{kcal}^{\mathrm{w}} \mathrm{wk}^{-1}\right)$ for data analysis. In the second part, an index of intensity is assessed from five distinct PA dimensions: the Vigorous Index combines the frequency and duration engaged in activities that involve large increases in breathing rate, heart rate, sweating and/or leg fatigue; the Walking Index reports the frequency and the duration of walking activities; the Moving Index measures the time spent each day in activities with movement while standing, including walking; the Standing Index evaluates the daily time spent in activities while standing without displacement; and the Sitting Index assesses time spent in the seated position for the day. Partial scores are multiplied by the specific weight factor to calculate the individual indexes and then summed to determine the Summary Index of activity (see http://dapatoolkit.mrc.ac.uk/documents/en/Yal/Yale_Physical_Activity_S urvey.pdf).

\subsection{YPAS-PT Translation and Cultural Adaptation}

The aim of the translation and cultural adaptation of the YPAS questionnaire is to obtain a final version of the instrument in the Portuguese language that is equally natural, acceptable and performs in the same way as the original YPAS. This process focused on WHO recommendations of cross-cultural and conceptual equivalence ${ }^{1}$ by following these steps: (1) Preparation: after permission from the authors, the questionnaire underwent a translatability review allowing drafted detailed instructions; (2) Translation: two independent certified bilingual translators performed independent forward translations to compile into a unified translation. This version was then given to an English native translator who performed a literal back-translation; (3) Harmonization: the expert panel, composed of health and sports experts and epidemiologists, performed a consensual version of YPAS-PT across all translations; (4) Cognitive Debriefing: the investigators conducted cognitive debriefing interviews with 10 older subjects, to ensure that this version of YPAS-PT was both culturally acceptable and contextually relevant to the target population; (5) Finalization: the researchers wrote the final version of YPAS-PT as a result of all the interactions described above.

\subsection{Validity}

The aim of this phase was to test the psychometric properties of YPAS-PT with a cross-reference standard, by accelerometry. Thus the criterion validity involved 96 participants $(75.2 \pm 7.1$ years; $64.6 \%$ women; BMI of $27.4 \pm 3.9$ kg. $\mathrm{m}^{-2}$ ) who used a uniaxial accelerometer (Actigraph, Model 7194) for 7 consecutive days on the right

1 Available at http://www.who.int/substance_abuse/research_tools/translation/en anterior superior iliac spine using an adjustable elastic belt [27]. They wore it all day, except while sleeping or doing activities that could damage the device (e.g. bathing, swimming). A daily log was provided to report the periods in which the accelerometer was worn and removed.

All logs completed by the subjects were checked and matched against the accelerometer data. Continuous sequences of 60 or more consecutive zero counts were deleted to distinguish the time when the accelerometer was not being worn. Individuals without a minimum of four days of valid recording ( $\geq 10 \mathrm{~h} /$ day or $\geq 3000$ counts/day) were excluded. The Freedson's cut-off points [27] were adopted in count ranges to allow the characterization of volume and intensity. Specifically, the volume of activity in: Activity Counts that represent a quantitative measure of activity over the time; Total Activity meaning the time of daily activity recorded; and, Total Physical Activity, considering total time spent in non-sedentary activities. Secondly, the intensity of activity was categorized as: Sedentary Activity, $<200$ counts per min (ct.min ${ }^{-1}$ ); Light Physical Activity, 201-1951 ct.min ${ }^{-1}$; Vigorous Physical Activity, > 5724 ct. $\mathrm{min}^{-1}$.

\subsection{Reliability}

Reliability of YPAS-PT was assessed by internal consistency and temporal stability. For internal consistency, 471 participants (74.9 \pm 6.5 years; $70.3 \%$ women) answered the YPAS-PT questionnaire. Temporal stability was verified through test-retest, conducted over a 2-week period, with 30 participants (71.9 \pm 3.9 years; $43.3 \%$ women) engaged in a PA community program.

\subsection{Data Management and Statistic Analysis}

Due to the negligible frequency of participation in vigorous and sedentary activities, it was decided to recode the YPAS-PT variables (time spent and EE) in: time in sedentary-to-light PA; time in moderate-to-vigorous PA; total PA time; EE in sedentary-to-light PA; EE in moderate-tovigorous PA; and Total EE in PA. Further, "minutes" (min) were used as the time unit to maintain consistency in the results of the different steps of validation.

All data were analyzed using IBM-SPSS 19.0. Descriptive statistics were obtained to summarize sample characteristics and PA patterns. Spearman correlation was applied to assess Criterion Validity. Accelerometer data were recorded using epochs of $1 \mathrm{~min}$, as commonly adopted [28, 29]. Stored data were downloaded using the software Actilife Lifestyle (v.3.2), and analyzed by MAHUFFE.exe software v.1.9.0.3 (www.mrc-epic.cam.ac.uk/). Energy expenditure was calculated using the regression equations and defined as Total $\mathrm{EE}(\mathrm{TEE})$ in $\mathrm{MET}=1.439008+\left(0.000795^{*}\right.$ ct.min- $\left.^{1}\right)$.

Reliability was tested through Cronbach's alpha to assess both internal consistency of total YPAS-PT checklist and YPAS-PT scores, and Intraclass Correlation Coefficient (ICC) to evaluate temporal stability. For the purposes of this study, coefficient values of $<0.20$ were taken to indicate poor agreement; $0.21-0.40$ represented fair agreement; $0.41-0.60$ 
were considered moderate or acceptable agreement; $0.61-$ 0.80 signified substantial agreement; and 0.81-1.0 represented almost perfect agreement [30].

\section{Results}

The final version of YPAS-PT resulted in a linguistic, conceptual and cultural equivalent questionnaire. As a consequence of the cognitive debriefing, in the "recreational activities" checklist, "Bocce" was replaced by the equivalent "petanque", and "Portuguese folk dances" were added to the "dance" field. "Hydro-gymnastics" was added to the "Exercise" checklist. The mean duration of the interview was $13 \mathrm{~min}$. A document with the guidelines for interviewers was produced.

At the end of the interview process it was noted that the training of the interviewers had a major role in assuring accurate YPAS-PT data as Intra-observer reliability (ICC) for each item ranged from 0.588 to 0.965 and from 0.938 to 0.998 for overall activity.
The validation process of the YPAS-PT involved 471 older adults $(74.9 \pm 6.5$ years). The majority were female $(73.3 \%)$, over half $(58 \%)$ were married and $84.3 \%$ lived in their own home. More than $60 \%$ of the participants had no formal education, or primary school education and the mean age for retirement was approximately 60 years. In general they spent $1280.5 \pm 1084.0$ min. $\mathrm{wk}^{-1}$ in PA, 953.6 $\pm 821.6 \mathrm{~min} . \mathrm{wk}^{-1}$ which included sedentary-to-light activity and $326.8 \pm 547.9 \mathrm{~min} . \mathrm{wk}^{-}$ ${ }^{1}$ in moderate-to-vigorous activities. Concerning TEE in PA, the total mean values were $4228.7 \pm 3819.1 \mathrm{kcal} \mathrm{wk}^{-1}$, which included $2601.9 \pm 2114.2 \mathrm{kcal} \mathrm{wk}^{-1}$ sedentary-to-light activity and $1686.7 \pm 2739.1 \mathrm{kcal} . \mathrm{wk}^{-1}$ moderate-to-vigorous activities. The mean values for the summary index were 53.1 \pm 29.3 , which consisted of $12.0 \pm 16.1$ for vigorous; $17.3 \pm 15.3$ for walking; $8.4 \pm 3.6$ for moving; $5.9 \pm 2.5$ for standing and $2.4 \pm 0.9$ for sitting indices (table 1 ). The mean values are higher than the median values, consistent with previous findings from Dipietro and colleagues [13] suggesting that these activity distributions are positively skewed.

Table 1. Physical Activity characteristics of older Portuguese by YPAS-PT $(n=471)$.

\begin{tabular}{|c|c|c|c|c|c|c|}
\hline & & Mean & \pm SD & Median & Minimum & Maximum \\
\hline \multicolumn{7}{|l|}{ YPAS-PT PA checklist } \\
\hline Total Time PA & $\min . \mathrm{wk}^{-1}$ & 1280.5 & \pm 1084.5 & 1035.0 & 0.0 & 7160.0 \\
\hline Time PA sedentary-to-light & $\min . w^{-1}$ & 953.6 & \pm 821.6 & 750.0 & 0.0 & 4310.0 \\
\hline Time PA moderate-to-vigorous & $\min . \mathrm{wk}^{-1}$ & 326.8 & \pm 547.9 & 130.0 & 0.0 & 5160.0 \\
\hline Total EE & kcal.wk ${ }^{-1}$ & 4288.7 & \pm 3819.1 & 3382.5 & 0.0 & 32710.0 \\
\hline EE sedentary-to-light & kcal.wk ${ }^{-1}$ & 2601.9 & \pm 2114.2 & 2130.0 & 0.0 & 12120.0 \\
\hline EE PA moderate-to-vigorous & kcal.wk ${ }^{-1}$ & 1686.7 & \pm 2739.1 & 720.8 & 0.0 & 28440.0 \\
\hline \multicolumn{7}{|l|}{ YPAS-PT PA dimensions } \\
\hline Summary index & score units & 53.1 & \pm 29.3 & 42.1 & 1.6 & 210.0 \\
\hline Vigorous index & score units & 12.0 & \pm 16.1 & 10.0 & 0.0 & 60.0 \\
\hline Walking index & score units & 17.3 & \pm 15.3 & 16.0 & 4.0 & 48.0 \\
\hline Standing index & score units & 5.9 & \pm 2.5 & 6.0 & 0.0 & 10.0 \\
\hline Sitting index & score units & 2.4 & \pm 2.0 & 2.0 & 1.0 & 4.0 \\
\hline Stair climbing & score units & 5.5 & \pm 11.3 & 2.0 & 0.0 & 150.0 \\
\hline
\end{tabular}

Note: The values are presented as mean, standard deviation $( \pm \mathrm{SD})$, median, minimum and maximum. Data from YPAS-PT checklist regarding a typical week was expressed as time in minutes engage in physical activities (Time PA) and energy expenditure (EE). Values are reported as "total physical activity" and two levels of different intensity ("sedentary-to-light" and "moderate-to-vigorous"). Data from the different PA dimensions of YPAS-PT are listed as units per day

In the Validity study, $64.6 \%$ of the participants were women, a percentage similar to that found in the Portuguese elderly population.

Table 2 highlights the main results. Based on YPAS-PT, the participants spent a total of $1256.4 \pm 850.3$ min. $\mathrm{wk}^{-1}$ (i.e., 179.5 min per day) in PA, $649.7 \pm 550$ min. $\mathrm{wk}^{-1}$ of which were for sedentary to light PA and $720.8 \pm 512.4$ min. $\mathrm{wk}^{-1}$ for moderate to vigorous PA. The PA levels allow a TEE of $43267.0 \pm 2723.0$ kcal.wk ${ }^{-1}$. Accelerometer data compared to YPAS-PT data reveals a lower time spent in PA and a higher estimation of EE. The accelerometer measured average time spent in PA was $838.91 \pm 78.8$ min. $\mathrm{wk}^{-1}$ (i.e., $119.8 \mathrm{~min}$ per day), $815.1 \pm 79.5$ min. $\mathrm{wk}^{-1}$ of which was for sedentary to light PA and $23.8 \pm 22.45$ min. $\mathrm{wk}^{-1}$ for moderate to vigorous $\mathrm{PA}$, and the total energy expenditure was $5020.4 \pm 1000.5 \mathrm{kcal} . \mathrm{wk}^{-1}$.

Table 3 shows the results of the criterion validity assessment. Spearman correlation coefficients between YPAS-PT and accelerometer parameters ranged from 0.232 to 0.408 , showing low-to-moderate pattern associations between self-reported (YPAS-PT) and measured accelerometer variables. As expected, we observed inverse correlations for the sitting index $(\mathrm{rho}=-0.305)$, with accelerometer parameters. 
Table 2. Physical Activity characteristics for Criterion Validity with a cross-reference standard by accelerometry $(n=96)$.

\begin{tabular}{|c|c|c|c|c|c|c|}
\hline & & Mean & $\pm S D$ & Median & Minimum & Maximum \\
\hline \multicolumn{7}{|l|}{ YPAS-PT PA checklist } \\
\hline Total Time PA & $\min . w^{-1}$ & 1256.4 & \pm 850.3 & 1005.0 & 120.0 & 3560.0 \\
\hline Time PA sedentary-to-light & $\min \cdot \mathrm{wk}^{-1}$ & 649.7 & \pm 550.9 & 500.0 & 10.0 & 2823.0 \\
\hline Time PA moderate-to-vigorous & $\min . w^{-1}$ & 720.8 & \pm 512.4 & 575.0 & 20.0 & 2570.0 \\
\hline Total EE & kcal.wk ${ }^{-1}$ & 4326.7 & \pm 2723.0 & 3495.8 & 120.0 & 12280.0 \\
\hline EE sedentary-to-light & kcal.wk ${ }^{-1}$ & 1418.9 & \pm 1051.8 & 1110.0 & 25.0 & 4620.0 \\
\hline EE PA moderate-to-vigorous & kcal.wk ${ }^{-1}$ & 2871.8 & \pm 2179.1 & 2290.0 & 42.5 & 10002.5 \\
\hline \multicolumn{7}{|l|}{ YPAS-PT PA dimensions } \\
\hline Summary index & score units & 59.2 & \pm 31.5 & 54.0 & 11.0 & 172.8 \\
\hline Vigorous index & score units & 17.8 & \pm 21.3 & 10.0 & 0.0 & 120.0 \\
\hline Walking index & score units & 23.5 & \pm 14.8 & 16.0 & 0.0 & 48.0 \\
\hline Moving index & score units & 9.1 & \pm 3.3 & 9.0 & 3.0 & 15.0 \\
\hline Standing index & score units & 6.3 & \pm 2.1 & 6.0 & 2.0 & 10.0 \\
\hline Sitting index & score units & 2.3 & \pm 0.8 & 2.0 & 1.0 & 4.0 \\
\hline Stair climbing & score units & 7.53 & \pm 16.8 & 3.0 & 0.0 & 150.0 \\
\hline \multicolumn{7}{|l|}{ ACCELEROMETERS } \\
\hline Total Time PA & $\min \cdot \mathrm{wk}^{-1}$ & 838.9 & \pm 78.8 & 840.9 & 675.4 & 990.0 \\
\hline Time PA sedentary-to-light $\left(<1951\right.$ ct.min $\left.^{-1}\right)$ & $\min . w^{-1}$ & 815.1 & \pm 79.5 & 813.8 & 572.1 & 982.8 \\
\hline Time PA moderate-to-vigorous (>1952 ct.min ${ }^{-1}$ ) & $\min \cdot \mathrm{wk}^{-1}$ & 23.8 & \pm 22.5 & 18.0 & 0.5 & 122.1 \\
\hline Total EE & kcal.w ${ }^{-1}$ & 5020.4 & \pm 1000.5 & 4833.8 & 2933.0 & 7349.8 \\
\hline
\end{tabular}

Note: The values are presented as mean, standard deviation $( \pm \mathrm{SD})$, median, minimum and maximum. Data from YPAS-PT checklist regarding a typical week was expressed as time in minutes engage in physical activities (Time PA) and energy expenditure (EE). Values are reported as "total physical activity" and two levels of different intensity ("sedentary-to-light" and "moderate-to-vigorous"). Data from the different PA dimensions of YPAS-PT are listed as units per day. Accelerometer data are calculated based on counts per minute $\left(\mathrm{ct}_{\mathrm{min}}{ }^{-1}\right)$ from the cut-off defined.

Table 3. Criterion Validity with a cross-reference standard, by accelerometry $(n=96)$.

\begin{tabular}{|c|c|c|c|c|}
\hline & Accelerometry & & & \\
\hline & Time sedentary to light & Time moderate to vigorous & Time total & EE Total \\
\hline & rho (p) & rho (p) & rho (p) & rho (p) \\
\hline \multicolumn{5}{|l|}{ YPAS-PT PA checklist } \\
\hline Time PA sedentary-to-light & & & & $-0.283(0.011)$ \\
\hline Time PA moderate-to-vigorous & & $0.396(0.001)$ & & \\
\hline Total Time PA & & $0.408(0.001)$ & & \\
\hline EE PA sedentary-to-light & & & & $-0.292(0.010)$ \\
\hline EE PA moderate-to-vigorous & & $0.363(0.001)$ & & \\
\hline Total EE PA & & $0.305(0.004)$ & & \\
\hline \multicolumn{5}{|l|}{ YPAS-PT PA dimensions } \\
\hline Vigorous index & & $0.250(0.014)$ & & \\
\hline Walking index & & $0.340(0.001)$ & $0.203(0.047)$ & \\
\hline \multicolumn{5}{|l|}{ Moving index } \\
\hline \multicolumn{5}{|l|}{ Standing index } \\
\hline Sitting index & & $-0.305(0.002)$ & & \\
\hline Summary index & & $0.331(0.001)$ & & \\
\hline Stair climbing & & & & $0.232(0.025)$ \\
\hline
\end{tabular}

Note: Data from YPAS-PT checklist regarding a typical week was expressed as time in minutes engage in physical activities (Time PA) and energy expenditure (EE). Values are reported as "total physical activity" and two levels of different intensity ("sedentary-to-light" and "moderate-to-vigorous"). Data from the different PA dimensions of YPAS-PT are listed as units per day. Accelerometer data are calculated based on counts per minute $\left(\right.$ ct.min $\left.^{-1}\right)$ the cut-off defined. 
Table 4. Physical Activity characteristics and results of temporal stability of YPAS-PT (test-retest over 2-week period) through Intraclass Corelation Coefficient (ICC), $(n=30)$.

\begin{tabular}{|c|c|c|c|c|c|c|c|c|}
\hline & & \multicolumn{3}{|c|}{$I^{\text {st }}$ moment (baseline) } & \multicolumn{3}{|c|}{$2^{\text {nd }}$ moment (retest) } & \multirow{2}{*}{ ICC } \\
\hline & & Mean & $\pm S D$ & Median & Mean & $\pm S D$ & Median & \\
\hline \multicolumn{9}{|l|}{ YPAS-PT PA checklist } \\
\hline Total Time PA & $\min \cdot \mathrm{wk}^{-1}$ & 1632.0 & \pm 701.8 & 1225.0 & 1664.2 & \pm 1279.7 & 1252.5 & $.925(.843, .964)$ \\
\hline Time PA sedentary-to-light & $\min . \mathrm{wk}^{-1}$ & 849.9 & \pm 701.8 & 719.2 & 855.3 & \pm 614.9 & 675.0 & $.887(.763, .946)$ \\
\hline Time PA moderate-to-vigorous & $\min . \mathrm{wk}^{-1}$ & 782.1 & \pm 685.0 & 685.0 & 808.9 & \pm 614.9 & 610.8 & $.911(.814, .958)$ \\
\hline Total EE & Kcal.wk ${ }^{-1}$ & 5771.8 & \pm 4247.6 & 4486.2 & 5787.6 & \pm 4310.2 & 4580.0 & $.921(.833, .962)$ \\
\hline EE sedentary-to-light & Kcal.wk ${ }^{-1}$ & 2412.1 & \pm 2020.5 & 1948.8 & 2416.0 & \pm 2121.6 & 2037.5 & $.903(.796, .954)$ \\
\hline EE PA moderate-to-vigorous & Kcal.wk ${ }^{-1}$ & 3357.6 & \pm 2607.7 & 3011.2 & 3371.6 & \pm 2940.0 & 2573.5 & $.899(.789, .952)$ \\
\hline YPAS-PT PA dimensions & & & & & & & & \\
\hline Summary index & units. $d^{-1}$ & 53.2 & \pm 17.8 & 50.9 & 56.8 & \pm 23.2 & 53.4 & $.650(.265, .834)$ \\
\hline Vigorous index & units. $^{-1}$ & 10.7 & \pm 10.9 & 10.0 & 10.8 & \pm 14.7 & 10.0 & $.645(.255, .831)$ \\
\hline Walking index & units. $d^{-1}$ & 20.8 & \pm 10.1 & 16.0 & 23.9 & \pm 12.1 & 240 & $.620(.201, .819)$ \\
\hline Moving index & units. $d^{-1}$ & 11.6 & \pm 3.0 & 12.0 & 11.6 & \pm 2.5 & 12.0 & $.632(.526, .893)$ \\
\hline Standing index & units. $d^{-1}$ & 8.3 & \pm 1.9 & 8.0 & 7.9 & \pm 2.2 & 8.0 & $.762(.500, .887)$ \\
\hline Sitting index & units. $d^{-1}$ & 2.1 & \pm 0.6 & 2.0 & 2.3 & \pm 0.8 & 2.0 & $.737(.448, .875)$ \\
\hline Stair climbing & units. $d^{-1}$ & 9.5 & \pm 9.9 & 6.0 & 10.7 & \pm 11.9 & 8.0 & $.851(.612, .912)$ \\
\hline
\end{tabular}

Note: The values are presented as mean, standard deviation $( \pm \mathrm{SD})$ and, median. Data from YPAS-PT checklist regarding a typical week was expressed as time in minutes engage in physical activities (Time PA) and energy expenditure (EE). Values are reported as "total physical activity" and two levels of different intensity ("sedentary-to-light" and "moderate-to-vigorous"). Data from the different PA dimensions of YPAS-PT are listed as units per day.

The reliability studies showed YPAS-PT checklist observed good internal consistency with a 0.835 Cronbach's alpha value however the YPAS-PT PA dimensions had unacceptable results, with a value of 0.454 .

Table 4 presents summary results of temporal stability. Concerning YPAS-PT checklist, the repeatability of measurements for total time PA showed an ICC of 0.925 (95\% CI $0.843-0.964)$ and for TEE an ICC of $0.921(95 \%$ CI 0.833 - 0.962). Regarding stability, substantial agreement was observed over time in YPAS-PT PA indices, ranging from an ICC of $0.620-851$ (95\% CI $0.201-0.819,95 \%$ CI $0.612-0.912$ ) respectively.

In general, participants spent $1632.0 \pm 1162.8 \mathrm{~min}$ (baseline) to $1664.2 \pm 1279.7 \mathrm{~min}$ (retest) per week in PA, $849.9 \pm 701.8 \mathrm{~min}$ (baseline) to $855.3 \pm 792.4 \mathrm{~min}$ (re-test) in sedentary-to-light activity and $782.1 \pm 592.8 \mathrm{~min}$ to $808.9 \pm 614.9$ minutes in moderate-to-vigorous activities, as reported in table 4 .

\section{Discussion}

Our aim was to adapt and validate the YPAS to the Portuguese culture and language. For these purposes, wellestablished methods were used to develop a Portuguese translation of the Yale Physical Activity Survey to ensure YPAS-PT. In accordance with WHO recommendations for cultural and conceptual relevance, the translation/backtranslation methodology and a questionnaire regarding PA patterns and levels, were administered by trained interviewers to enable a high level of comprehension and relevance of questions among the older adult participants.

Our results compare favorably with previous validations of YPAS. As shown in table 3, low-to-moderate criterion validity was documented in most of the PA variables (rho 0.232 to 0.408 ) when testing the relationship between the recalled activity (YPAS-PT) and the measured parameters (accelerometers). Shephard [2] stresses the importance of this type of validation, as it can indicate the degree to which YPAS-PT is able to quantify weekly PA. In this study, a moderate correlation between total time in PA between YPAS-PT and accelerometry (rho 0.408; p $\leq$ 0.001) was measured which is consistent with previous correlations between accelerometry and PA questionnaires, which ranged between 0.28 and 0.52 [14, 21].

This study assessed PA levels using YPAS-PT and accelerometry among older adults. The questionnaire aimed to accurately estimate EE, including the light intensity activities, such as ADLs that contribute to total PA time in this population. The correlation between YPAS-PT and accelerometry may not have been sensitive to a number of factors within older populations. For example, the reported time in moderate to vigorous PA $\left(1256.4 \pm 850.3\right.$ min. $\left.^{-1}\right)$ and the one measured by the accelerometer $(838.9 \pm 78.8$ min. $\mathrm{wk}^{-1}$ ) could be explained by the cutoff points used from the accelerometry data recorded (Table 2). The points were based on the equations by Freedson et al [27] and might be set too high to capture the PA characteristic of the elderly [31]. Of course it is possible that participants may be overestimating moderate to vigorous PA (in particularly vigorous PA) in their YPAS responses, since the answers are given based on their perceived exertion.

When Kolbe-Alexander et al [26] compared two methods of measuring PA in older South African adults (YPAS and IPAC vs. accelerometry), better correlation coefficients were found in the criterion validity for men. The authors reported that women spent less time in vigorous activities (IPAC), and household EE (measured by YPAS) gave the most contribution to total weekly EE. They argued that lower intensity such as household activities are unlikely to be accurately recorded by the accelerometer (MTI Actigraph), which could explain the lower criterion validity than the one found in men. The sample for this study consisted of $64.6 \%$ 
women, with an average age (75.2 \pm 7.1 years) higher than most of the YPAS validation studies, which may have a special significance in the results obtained. Further, lowerintensity activities, such as ADLs often comprise irregular upper limb activity as opposed to ambulatory activity such as walking which has previously been identified as a limitation in using accelerometry by Basset et al [31]. The use of another method to validate YPAS-PT such as portable energy consumption device may delineate effects noted in this study related to age, gender and intensity with energy consumption estimations.

YPAS-PT validity results (table 3) are comparable to previously published data on PA questionnaires [8, 13, 18-21, 23, 32]. Martín et al [22] reported similar results, with the Spanish version of YPAS, when compared to a test based on submaximal performance in a group of Spanish-speaking healthy older women ( $\mathrm{rho}=0.349, \mathrm{p}<0.02$ ).

YPAS-PT performed similarly in terms of reliability in comparison to other studies [8, 13, 17-19, 31]. YPAS-PT revealed statistical significance in temporal stability, resulting in an "almost perfect agreement" range from baseline and re-test of application for the YPAS-PT checklist (ICC of $0.887-0.925 ; 95 \%$ CI $0.763-0.946,0.843-0.946$, respectively), and "moderate to good agreement" regarding YPAS-PT PA dimensions (ICC of $0.620-0.851 ; 95 \%$ CI $0.201-0.819,0.612-0.912$, respectively) (table 4$)$. In the validation of the original survey, DiPietro et al. [13] reported correlation coefficients between the two administrations for the YPAS summary indices ranging from $0.42(\mathrm{p}=0.002)$ to $0.65(p=0.001)$ and found that high-intensity exercise-related behaviors repeated much better than more common lower intensity activities, surmising that these may be more habitual and thus, more difficult to recall accurately. They also argued that it was difficult to assess whether the low agreement or significant mean differences in scores between survey administration are a result of the difficulty of recalling, or actual changes in behavior, or if the subjects paid closer attention to their activity after the first administration. From the first to the second application, the average values of all variables are indeed lower [13] than the ones registered by YPAS-PT, where we noted a small increase in all average values. This reinforced our conviction that, from the first application, participants were enthusiastic about the need to be physically active and seemed to enjoy reporting their activities in a typical week. This commitment may have influenced behavior change and assisted in the good results we obtained. Also, perhaps due to the interest in participation, the authors of this study cannot identify any issue with the recall of activity among participants. It may also be reflective of this study's sample and exclusion criteria may have skewed recall results.

The reliability of YPAS has been tested by other researchers in different populations and countries: De Abajo et al. [8] verified the reliability of the Spanish version for all variables of YPAS checklist and YPAS summary indices, with the exception of standing. Later on, Pennathur et al. [24], observed "moderate to good" reliability for PA assessment in older Mexican-American adults in weekly EE and vigorous activity index score. In South Africa, KolbeAlexander et al. [26] observed that YPAS reliability ranged from $r=0.44$ to 0.80 for men and $r=0.59$ to 0.99 for women ( $\mathrm{p}<0.001)$. According to these authors, YPAS captures predominantly low-intensity activities that are usual to older adults (especially in women), who might have resulted in a better recall. In YPAS-PT temporal stability study, although the majority of participants were males $(56.7 \%)$, they had an average age (71.9 \pm 3.9 years) higher than the studies reported, with predominant participation in sedentary to moderate PA, i.e., low-intensity activities. Furthermore, the authors in this study recoded the variables, time spent and $\mathrm{EE}$, into sedentary-to-light and moderate-to-vigorous, which may have assisted in sensitivity to measure the levels of physical activity.

The YPAS is a questionnaire specifically designed to measure physical activity of older adults with considerable intuitive appeal because it captures the types and intensities of activities that are common among the elderly that are typically excluded from other instruments [14, 32]. The main results of YPAS-PT, as shown in table 1, estimated an average magnitude of weekly EE in PA of $4288.7 \pm 3819.1 \mathrm{kcal}^{\mathrm{w}} \mathrm{wk}^{-1}$, by older Portuguese adults, that were lower than those reported by DiPietro et al. [13] in their original YPAS study, and in older adults in Connecticut, USA, who spent an estimated average of $6739.7 \pm 4267.2$ to $7613.2 \pm 4502.1 \mathrm{kcal}^{-\mathrm{wk}^{-1}}$ during two administrations of YPAS. Schuler et al. [18] reported values of $8000 \mathrm{kcal} \cdot \mathrm{wk}^{-1}$

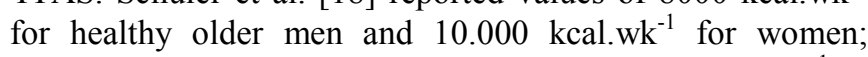
Pannathur et al. [19] also found $6750.0 \pm 3292.0 \mathrm{kcal}^{\mathrm{w}} \mathrm{wk}^{-1}$ in older Mexican American adults and De Abajo et al. [8] estimated similar values for Spanish older adults (6335 kcal. $\mathrm{wk}^{-1}$ for 38 men and 7314 kcal.wk $\mathrm{wh}^{-1}$ for women).

Portuguese older population, according to the National survey of PA [4] were mostly sedentary (55\% and $72 \%$ of the men and women, respectively), which is in agreement with the data shown in our study and those reported by Young et al. [14] with an average weekly EE in PA of $3285{\mathrm{kcal} . \mathrm{wk}^{-1}}^{-1}$ for African-American older adults (data from 27 men and women combined) who initially were classified as sedentary.

When comparing the magnitude of total time spent in PA in a week (table 1), the older Portuguese participants spent $1280.5 \pm 1084.5$ min. $\mathrm{wk}^{-1}\left(21.3 \mathrm{~h} . \mathrm{wk}^{-1}\right)$ in PA, less than the values obtained by DiPietro et al. [13] in the validation of the original survey (30.6 and $34.3 \mathrm{~h} \cdot \mathrm{wk}^{-1}$ ). Even so, the mean time spent on sedentary-to-light PA was 953.6 \pm 821.6 min. $\mathrm{wk}^{-1}\left(15.9 \pm 13.7 \mathrm{~h} . \mathrm{wk}^{-1}\right)$ and $326.8 \pm 547.9 \mathrm{~min} . \mathrm{wk}^{-1}$ $\left(5.5 \pm 9.1\right.$ h. $\left.\mathrm{wk}^{-1}\right)$. Although it would be ideal to see more PA among the Portuguese population, on average, they respect the PA guidelines [30], spending $183 \mathrm{~min}^{-1} \mathrm{~d}^{-1}$ of time in PA, 46.7 min. $\mathrm{d}^{-1}$.

Concerning PA dimensions, the mean scores of the indices were $53.1 \pm 29.3$ for the summary index and $12.0 \pm 16.1$ for the vigorous, $17.3 \pm 15.3$ for the walking, $8.4 \pm 3.6$ for the moving, $5.9 \pm 2.5$ for the standing and $2.4 \pm 0.9$ for the sitting ones. These results are comparable to those found by Martín et al [22]. 
Accurate measurements of habitual PA is fundamental to both the epidemiological study of relations between PA \& health and the recommendations of an appropriate pattern of PA to maintain good health. It is important that development of standardized instruments record all activity, particularly the low-intensity activities typical of sedentary societies [2]. Although we cannot compare our results with those of the Portuguese survey recorded by IPAC $[4,10]$, it seems that YPAS could be more appropriate to measure PA in older adults, as it includes activities that are common among older adults, particularly light-intensity activity, which is often excluded from other physical activity assessment tools [14], and could detect changes in activity after a physical activity intervention [7, 14, 32]. Kolbe-Alexander and colleagues [26] compared the YPAS and the short IPAQ to measure PA in South African older adults and found both provide reasonably reliable estimates of physical activity in older adults in this community. The reliability of the specific YPAS domains, however, such as housework and yard work, was more robust than the vigorous and moderate indices of the IPAQ. Subsequently, they recommend the YPAS for research studies, particularly those that wish to quantify the types of physical activities habitually performed by older adults. Our results extend data obtained and can contribute to better establish the internal validity of the questionnaire, showing good internal consistency $(\alpha=0.835)$ regarding YPAS-PT checklist. Further studies that reproduce the YPAS-PT are required for obtaining further evidence of the reliability of this tool within Portuguese-speaking populations.

To our knowledge, this is the first validation of a Physical Activity questionnaire for Portuguese older adults. It is especially relevant since it updates and supports the knowledge of PA levels in this population allowing comparisons within European or International contexts. Nevertheless, some limitations should be considered when interpreting our findings. Firstly, because the sub-samples of participants, namely those included in the temporal stability study, are smaller, compared to the main study sample. Secondly, because collecting PA data from questionnaires should be conservative due to memory recall. Nevertheless, data have been recorded as part of an extensive protocol, allowing to confirm the reliability of the collected information. Additionally, we intend that YPAS-PT questionnaire should be used in large scale of older populations, because it reduces the effect of hypothetic bias.

\section{Conclusion}

The process of validation of YPAS-PT demonstrates acceptable validity and reliability to measure physical activity levels in the older-adult Portuguese population. This study was able to use the YPAS-PT to record low-intensity activities typical of sedentary behavior, which is often excluded from other PA assessment tools, and ascribe consistent biological meaning to terms such as light, moderate, and heavy exercise typical of aging societies.

The assessment and definition of PA patterns sustained from an ecological perspective allowed results from YPAS-PT to capture typical daily patterns specific to aging populations.

Subsequently, YPAS-PT is recommended for research studies that have designed intervention strategies for specific needs of the older population that wishes to quantify the types of physical activities habitually performed by older adults.

We have concluded that the Portuguese version of YPAS (i.e., YPAS-PT) is a valid instrument to assess PA patterns of elderly Portuguese for research and community interventions.

\section{Acknowledgments}

The authors would like to thank the project staff and the study participants, who invested their time and energy in our research.

This article was presented at the $67^{\text {th }}$ Annual Meeting of the Gerontological Society of America, November 2014, Washington, DC.

\section{References}

[1] World Health Organization. Global Recommendations on Physical Activity for Health. Geneva: WHO Press. 2010. ISBN 9789241599979.

[2] Shephard RJ. Limits to the measurement of habitual physical activity by questionnaires. Br J Sports Med 2003; 37(3): 197206.

[3] Instituto Nacional de Estatística. Projecções de População Residente em Portugal 2008-2060. INE, IP. Lisboa. 2009. ISBN 978-989-25-0037-9.

[4] Baptista F, Silva AM, Santos DA, Mota J, Santos R, Vale S, et al. Livro Verde da Atividade Física. IDP, I. P. Lisboa, 2011. ISBN: 978-989-8330-02-4.

[5] Chodzko-Zajko WJ, Proctor DN, Fiatarone Singh MA, Minson CT, Nigg CR, et al. Exercise and Physical Activity for Older Adults (Position Stand). Med Sci Sports Exerc 2009; 41(7): 1510-30. DOI: 10.1249/MSS.0b013e3181a0c95c.

[6] Kowalski K, Rhodes R, Naylor PJ, Tuokko H, Macdonald S. Direct and Indirect Measurement of Physical Activity in Older Adults: A Systematic Review of the Literature. Int J Behav Nutr Phys Act 2012; 9: 148-169.

[7] Strath SJ, Kaminsky LA, Ainsworth BE, Ekelund U, Freedson PS, Gary RA, et al. Guide to the Assessment of Physical Activity: Clinical and Research Applications: A Scientific Statement From the American Heart Association. Circulation. 2013; 128 : 2259-2279; DOI: 10.1161/01.cir.0000435708.67487.da.

[8] De Abajo S, Larriba R, Marquez S. Validity and Reliability of the Yale Physical Activity Survey in Spanish Elderly. J Sports Med Phys Fitness 2001; 41(4): 479-485.

[9] Craig C, Marshall AL, Sjöström M, Bauman AE, Booth ML, Ainsworth $\mathrm{BE}$, et al. International physical activity questionnaire: 12-country reliability and validity. Med Sci Sports Exerc. 2003; 35(8): 1381-95. DOI:10.1249/01.MSS.0000078924.61453.FB. 
[10] Baptista F, Santos DA, Silva AM, Mota J, Santos R, Vale S, et al. Prevalence of the Portuguese Population attaining sufficient physical activity. Med Sci Sports Exerc 2012; 44(3): 466-473. 2012. DOI: 10.1249/MSS.0b013e318230e441.

[11] Tomioka K, Iwamoto J, Saeki K, Okamoto N. Reliability and validity of the International Physical Activity Questionnaire (IPAQ) in elderly adults: the Fujiwarakyo Study. J Epidemiol 2011; 21(6): 459-465. doi:10.2188/jea.JE20110003.

[12] Heesch KC, Van Uffelen JG, Hill RL, Brown WJ. What do IPAQ questions mean to older adults? lessons from cognitive interviews. Int $J$ Behav Nutr Phys Act 2010, 7: 35 http://www.ijbnpa.org/content/7/1/35.

[13] DiPietro L, Caspersen CJ, Ostfeld AM, Nadel ER. A Survey for Assessing Physical Activity Among Older Adults. Med Sci Sports Exerc 1993; 25(5): 628-642.

[14] Young D, Jee S, Appel L. A Comparison of the Yale Physical Activity Survey With Other Physical Activity Measures. Med Sci Sports Exerc 2001; 33 (6): 955-961. doi: 01959131/01/3360.

[15] Bonnefoy, M, Normand S, Pachiaudi C, Lacour R, Laville M, Kostka T. Simultaneous Validation of Ten Physical Activity Questionnaires in Older Men: A Doubly Labeled Water Study. J Am Geriatr Soc 2001; 49(1): 28-35.

[16] Prince S, Adamo K, Hamel M, Hardt J, Gorber S, Tremblay M. A Comparison of Direct Versus Self-Report Measures for Assessing Physical Activity in Adults: a Systematic Review. Int J Behav Nutr Phys Act 2008; 5: 56-80. doi: 10.1186/14795868-5-56.

[17] Starling RD, Matthews DE, Ades PA, Poehlman ET. Assessment of Physical Activity in Older Individuals: a Doubly Labeled Water Study. J Appl Physiol 1999; 86(6): 2090-2096.

[18] Schuler PB, Richardson MT, Ochoa P, Wang MQ. Accuracy and Repeatability of the Yale Physical Activity Survey in Assessing Physical Activity of Older Adults. Percept Mot Skills 2001; 93(1): 163-177.

[19] Pennathur A, Magham R, Contreras LR, Dowling, W. Daily Living Activities in Older Adults: Part II-Effect of Age on Physical Activity Patterns in Older Mexican American Adults. Int J Ind Ergon 2003; 32(6): 405-418. doi: 10.1016/S01698141(03)00099-4.

[20] Kruskall LJ, Campbell, WW, Evans WJ. The Yale Physical Activity Survey for Older Adults: Predictions in the Energy Expenditure Due to Physical Activity. J Am Diet Assoc 2004; 104: 1251-1257. doi: org/10.1016/j.jada.2004.05.207.

[21] Donnaire-Gonzalez D, Gimeno-Santos E, Serra I, Roca J, Balcells E, Rodríguez E, et al. Validation of the Yale Physical Activity Survey in Chronic Obstructive Pulmonary Disease Patients. Arch Bronconeumol 2011; 47: 552-560.
[22] Martín V, Ayán C, Molina AJ, Alvarez MJ, Varela S, Cancela JM. Correlation Between the Yale Physical Activity Survey (YPAS) and Submaximal Performance-Based Test: a Study in a Population of Elderly Spanish Women. Arch Gerontol Geriatr 2012; 55(1): 31-34. doi: 10.1016/j.archger.2011.06.016.

[23] Katz JN, Perez MT, Niu NN, Dong Y, Brownlee S, Elma SA, et al. Development and Validation of a Spanish Translation of the Yale Activity Questionnaire. BMC Musculoskelet Disord 2014; 15: 120. doi: 10.1186/1471-2474-15-120.

[24] Pennathur A, Magham R, Contreras Lr, Dowling W. TestRetest Reliability of Yale Physical Activity Survey Among Older Mexican American Adults: a Pilot Investigation. Exp Aging Res 2004; 30(3): 291-303. doi: $10.1080 / 03610730490447912$.

[25] Semanik P, Lee J, Manheim L, Dipietro L, Dunlop D, Chang RW. Relationship Between Accelerometer-Based Measures of Physical Activity and the Yale Physical Activity Survey in Adults with Arthritis. Arthritis Care Res 2011; 63 (12): 17661772. doi:10.1002/acr.20644.

[26] Kolbe-Alexander TL, Lambert EV, Harkins JB, Ekelund U. Comparison of Two Methods of Measuring Physical Activity in South African Older Adults. J Aging Phys Act 2006; 14: 98114.

[27] Freedson Ps, Melanson E, Sirad J. Calibration of the Computer Science and Applications. Inc. Accelerometer. Med Sci Sports Exerc 1998; 30(5): 777-81. doi: 10.1097/00005768199805000-00021.

[28] Pruitt LA, Glynn NW, King AC, Guralnik JA, Aiken EK, Miller G, et al. Use of Accelerometry to Measure Physical Activity in Older Adults at Risk for Mobility Disability. J Aging Phys Act 2008; 16(4): 416-434. doi: 10.1249/00005768-199305000-0001

[29] Copeland JL, Esliger DW. Accelerometer Assessment of Physical Activity In Active, Healthy Older Adults. J Aging Phys Act 2009; 17(1): 17-30.

[30] Szklo M, Nieto J. Epidemiology: Beyond the Basics. $2^{\text {nd }}$ ed. Jones and Bartlett Publishers, Inc., 2007. ISBN- 13: 978-07637-2927-1.

[31] Bassett Jr DR, Ainsworth BE, Swartz AM, Strath SJ, O'Brien WL, King GA. Validity of Four Motion Sensors in Measuring Moderate Intensity Physical Activity. Med Sci Sports Exerc 2000; 32(9): S471-480.

[32] Harada ND, Chiu V, King AC, Stewart AL. An Evaluation of Three Self-Report Physical Activity Instruments for Older Adults. Med Sci Sports Exerc 2001; 33: 962-970. 